\title{
Prediction of Betavoltaic Battery Output Parameters Based on SEM Measurements
}

\author{
E.B. Yakimovi,2,*, M.A. Polikarpov³, A.A. Krasnov² \\ ${ }^{1}$ Institute of Microelectronics Technology RAS, 6, Institutskaya st., 142432 Chernogolovka, Russia \\ 2 University of Science and Technology MISiS, 4, Leninskiy prosp., 119040 Moscow, Russia \\ ${ }^{3}$ National Research Center Kurchatov Institute, 1, Akademika Kurchatova sq., 123182 Moscow, Russia
}

(Received 04 August 2016; published online 23 December 2016)

\begin{abstract}
The approach for the prediction of betavoltaic battery output parameters based on EBIC investigations of semiconductor converters of beta-radiation energy into electric power is presented. Using this approach the parameters of battery based on porous Si are calculated. These parameters are compared with those of battery based on a planar Si $p-n$ junction.
\end{abstract}

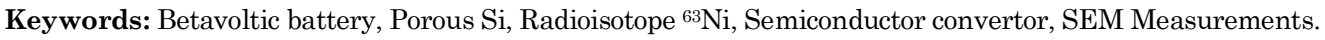

DOI: $10.21272 /$ jnep.8(4(2)).04062

PACS numbers: 84.60. - h, 85.30. - z

\section{INTRODUCTION}

In betavoltaic batteries energy of nuclear radiation is directly converted to electric power using a semiconductor convertor. Radioactive isotopes are rather expensive; therefore for a successful design optimization of effective converters, methods for their testing before the radioactive film deposition should be developed. A promising idea is to use for this purpose e-beam of SEM, which could imitate the beta-radiation. This idea have been realized in a few works (for example, see $[1,2])$ and beam energy of $17 \mathrm{keV}$ corresponding to the mean energy of electrons emitted by the ${ }^{63} \mathrm{Ni}$ isotope was usually used for the beta-radiation imitation. However, elastic and inelastic scattering and absorption inside the ${ }^{63} \mathrm{Ni}$ film modify significantly the spectrum of electrons emitted from ${ }^{63} \mathrm{Ni}$ atoms. Even if this spectrum is measured, its knowledge is insufficient to calculate the induced by beta-radiation current because the beta particle angle distribution should be also known. Trajectories of electrons emitted from the ${ }^{63} \mathrm{Ni}$ film are distributed in a wide range of angles and this distribution depends on the film thickness and electron energy. Besides, electron range is proportional to $E_{b} b^{1.75}$, where $E_{b}$ is the e-beam energy. Thus, for the conducting materials with a small diffusion length the mean energy is not a suitable parameter to predict the collection efficiency. For the reasons mentioned the best approach for induced current calculations is to use the depth dependence of energy losses (depth-dose dependence) inside a semiconductor instead of the energy spectrum and angle distribution of particles emitted from the film. Such depth-dose dependences determining the depth dependences of excess carrier generation rate in $\mathrm{Si}, \mathrm{SiC}$ and $\mathrm{GaN}$ for beta particles emitted from ${ }^{63} \mathrm{Ni}$ films of different thickness were calculated in $[3,4]$ by the Monte-Carlo program taking into account an isotropic emission of radiation, the full energy spectrum of electrons emitted from the ${ }^{63} \mathrm{Ni}$ isotopes and inelastic and elastic scattering processes inside a $\mathrm{Ni}$ film. It was shown that a decay of these functions with a depth is close to exponential one, i.e. it differs qualitatively from that for monoenergetic electron beam perpendicular to the surface, which is described by the Gaussian function. Therefore, it is impossible to imitate the beta particle generation function using one or a few e-beam energies and other approaches for betaradiation imitation using SEM should be developed.

The approach based on calculations of induced current by a convolution of the depth-dose dependence with the collection probability obtained from the EBIC measurements was proposed in $[5,6]$. The induced current generated by beta-radiation is a key parameter in this approach. Other output parameters of converter can be also obtained by direct measurements in SEM [6]. For Si planar structures the current induced by beta-radiation was calculated in [5-7]. The thicknesses of the SiC and silicon wafers are usually varied in the range from $200 \mu \mathrm{m}$ to $400 \mu \mathrm{m}$, where only the top $15 \mu \mathrm{m}$ thick layer is the active functioning region. To maximize the battery output power density two approaches can be used. The first one consists in thinning a semiconductor structure to a thickness of a few tens of microns [8] and constructing the battery by stacking thinned-down converters and $\mathrm{Ni}$ films together. The other one is to use 3-D structures, for example, based on porous $\mathrm{Si}$, to increase the effective converter surface [9-11]. Such 3-D structures allow converting beta particle energy more efficiently in addition to an increase of effective surface area of elements [10]. However estimations of such converter efficiency were not carried out up to now.

In the present paper the induced current was calculated for the semiconductor convertor based on porous Si. The results obtained were compared with those obtained for planar Si-based structures.

\section{INDUCED CURRENT CALCULATIONS}

As shown in [12], the current induced in semiconductor structures by e-beam irradiation Ic can be calculated as a convolution of depth-dose function $h(z)$ with the collection probability $\psi(z)$, i.e. the probability that a minority carrier generated at a depth $z$ below the surface will be collected by a collector.

\footnotetext{
*yakimov@iptm.ru
} 


$$
I_{c}=\int_{0}^{d} h(z) \psi(z) d z
$$

Thin ${ }^{63} \mathrm{Ni}$ film with the specific activity of $10 \mathrm{Ci} / \mathrm{g}$ is taken as a radioactive source because ${ }^{63} \mathrm{Ni}$ is considered as the most promising source for long-living radioisotope-based batteries. The depth-dose dependence for $\mathrm{Si}$ irradiated with beta particles from such film was calculated in [3] as a function of radioactive film thickness. For thicknesses of Ni film in the micrometer range this dependence can be approximated with the exponential function $h(z)=A \times \exp (-z / 3.45)$, where $A$ is the parameter depending on the ${ }^{63} \mathrm{Ni}$ film thickness and specific activity, $\mathrm{z}$ is expressed in microns and the characteristic decay length is of $3.45 \mu \mathrm{m}$. This dependence is saturated for a thickness of about $3 \mu \mathrm{m}$, therefore the optimal thickness of $\mathrm{Ni}$ film is in the range between 1 and $3 \mu \mathrm{m}$ and its choice is determined by a compromise between the price of ${ }^{63} \mathrm{Ni}$ film and the battery output power. In the case of porous $\mathrm{Si}$ the pore diameter should be also taken into account.

The collection probability can be obtained by fitting the measured dependence of collected current in the EBIC mode on beam energy [13]. For planar Si based structures such procedure allows to obtain the collection probability with a rather high precision. It is not so easy to carry put such measurements on porous $\mathrm{Si}$ structures, therefore in the present paper the collection probability obtained using such procedure for the planar Si p-n structure studied in [14] was used in calculations. This probability can be approximated by a linear dependence in the upper $0.3 \mu \mathrm{m}$ thick $p$-region changing from 0.45 at the surface to 1 at the top depletion region boundary. Inside the depletion region and to a depth of $10 \mu \mathrm{m}$ in $n$-Si it is close to 1 .

It should be noted that, as shown in [6], for semiconductors with the small diffusion length and the large depletion region width such approach for the collection probability determination is not so straightforward due to a necessity to take into account the excess carrier recombination inside the depletion region. However, in Si-based structures the diffusion length is usually rather large and the problem could arise only for $p$ - $i$ - $n$ structures with very wide $i$-region that is not necessary for betavoltaic applications.

\subsection{Assumed Structure Design}

Calculations were carried out for porous Si with the realistic parameters presented in [11], i.e. the pore radius is of $3 \mu \mathrm{m}$, the pore density $N=10^{6} \mathrm{~cm}^{2}$, the pore depth is of $200 \mu \mathrm{m}$. It is assumed that $0.3 \mu \mathrm{m} p-n$ junction is formed inside every pore and $1 \mu \mathrm{m}$ thick ${ }^{63} \mathrm{Ni}$ film with the specific activity of $10 \mathrm{Ci} / \mathrm{g}$ is deposited on it uniformly on the entire depth. The porous structure is approximated by an array of hexagonal units perpendicular to the surface with a cylindrical pore inside every unit (Fig. 1). Thus, the values $r=3 \mu \mathrm{m}$ and $d=10 \mu \mathrm{m}$ were used. Because of periodicity, it is sufficient to calculate the induced current for unit cell. To simplify the calculations, the hexagonal unit cell is approximated with the equivalent circle of area $\pi R^{2}=1 / N$, so that the total volume of this array of cylinders is equal to the crystal surface multiplied by a depth of $200 \mu \mathrm{m}$. Irradiation from Ni film covering the pore inside the unit cell and from $\mathrm{Ni}$ films covering 6 adjacent pores was taken into account under calculations.

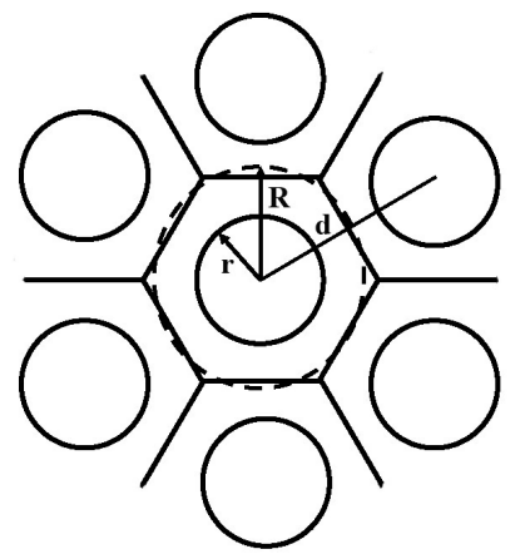

Fig. 1 - Schematic illustration of unit cell and its approximation with a cylinder

\section{THE RESULT OBTAINED}

For the structure studied and assumed parameters of ${ }^{63} \mathrm{Ni}$ film the calculated density of the induced current, which is equal to the total short circuit current divided by the surface area, is of $3.9 \mu \mathrm{A} / \mathrm{cm}^{2}$. Such approach allows to predict the parameters of semiconductor converter based on porous $\mathrm{Si}$ as a function of their radius and density that can be used for their optimization. An example of such calculations is presented in Fig. 2, where the induced current as a function of pore density is shown for the fixed pore radius of $3 \mu \mathrm{m}$. It is seen that as expected, the induced current increases with the pore density practically linear, however, it start to decrease, when the pore diameter approaches $\mathrm{d}$. The maximum induced current is about $5 \mu \mathrm{A} / \mathrm{cm}^{2}$ at the pore density of $1.5 \cdot 10^{6} \mathrm{~cm}^{2}$.

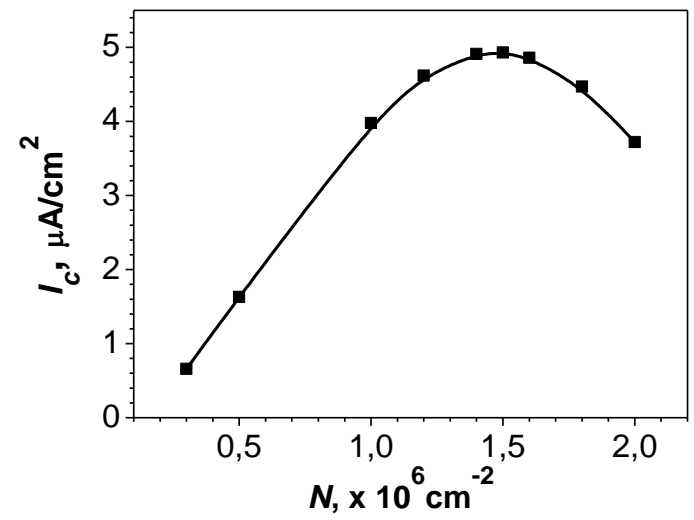

Fig. 2-Dependence of induced current on pore density for pore radius of $3 \mu \mathrm{m}$

\subsection{Comparison with Planar Structures}

For the planar Si p-n diode covered with $3 \mu \mathrm{m}$ thick ${ }^{63} \mathrm{Ni}$ film with the specific activity of $10 \mathrm{Ci} / \mathrm{g}$ the induced current density was calculated in [6], where a value of $110 \mathrm{nA} / \mathrm{cm}^{2}$ was obtained. For the $1 \mu \mathrm{m}$ thick film the 
calculated induced current density is equal to $78 \mathrm{nA} / \mathrm{cm}^{2}$, i.e. 50 times lower than that for the porous Si based structure. The total effective area of pores in $1 \mathrm{~cm}^{2}$ is about $38 \mathrm{~cm}^{2}$, i.e. in porous $\mathrm{Si}$ structure with the parameters used in the calculations the efficiency of beta-radiation utilization increases 1.3 times in comparison with the planar structure.

However, it should be taken into account that at $\mathrm{Si}$ thickness of $9 \mu \mathrm{m}$ it absorbs about $95 \%$ of betaradiation energy. Thus, a stack of 10 planar structures with a total thickness of $200 \mu \mathrm{m}$ produces the current only 5 times smaller than the corresponding structure based on porous Si. Besides, in a planar structure only $50 \%$ emitted electrons are utilized, while in the stacking thinned-down devices about all of them can be used that increases two times more the induced current. Thus, the induced current in this device can be only 2.5 times lower than that in the porous Si based structure of the same volume. It should be also taken into account that the technology of planar structures is much simpler and therefore the open circuit voltage and filling factor for them should be higher than those for deep

\section{REFERENCES}

1. M.V.S. Chandrashekhar, C.I. Thomas, H. Li, M.G. Spencer, A. Lal, Appl. Phys. Lett. 88, 033506 (2006).

2. B. Ulmen, P.D. Desai, S. Moghaddam, G.H. Miley, R.I. Masel, J Radioanal Nucl. Chem. 282, 601 (2009).

3. V.N. Pavlov, V.Ya. Panchenko, M.A. Polikarpov, A.A. Svintsov, E.B. Yakimov, J. Surface Investig. X-ray, Synchrotron Neutron Techniq. 7(5), 852 (2013).

4. S.I. Zaitsev, V.N. Pavlov, V.Ya. Panchenko, M.A. Polikarpov, A.A. Svintsov, E.B. Yakimov, J. Surface Investig. X-ray, Synchrotron Neutron Techniq. 8(5), 843 (2014).

5. M.A. Polikarpov, E.B. Yakimov, Semiconductors 49(6), 746 (2015).

6. E.B. Yakimov, Appl. Radiat. Isot. 112, 98 (2016).

7. A.A. Krasnov, S.A. Legotin, V.N. Murashev, S.I. Didenko, O.I. Rabinovich, S.Y. Yurchuk, Y.K. Omelchenko, E.B. Yakimov and V.V. Starkov, IOP Conf. Series: Materi- pore or trench structures. Besides, it is not so easy to deposit Ni layer with the homogeneous thickness along their length. Such factors can essentially decrease the efficiency of converters based on porous Si. For these reasons it seems that the stacking thinned-down structures are more promising for the effective semiconductor converter than those based on porous Si.

\section{CONCLUSION}

Thus, the induced current in semiconductor convertor of beta-radiation energy based on porous $\mathrm{Si}$ is calculated. The obtained values are compared with those for the convertor based on the stacking thinned-down planar Si $p$ - $n$ junctions. It is shown that the output parameters of the stacking thinned-down devices can be comparable or even higher than those of devices based on porous $p$ - $n$ junctions.

\section{AKNOWLEDGEMENTS}

The work was partially supported by the Russian Foundation for Basic Research (grant 14-29-04056).

als Science and Engineering. 110, 012029 (2016).

8. S. Tin, A. Lal, PowerMEMS, Washington DC, 189 (2009).

9. W. Sun, N.P. Kherani, K.D. Hirschman, L.L. Gadeken, P.M. Fauchet, Adv. Mater. 17(10), 1230 (2005).

10. T. Wacharasindhu, B.R. Nullmeyer, J.W. Kwon, J.D. Robertson, A.Y. Garnov, J. Microelectromech. Syst. 23(1), 56 (2014)

11. V.V. Starkov S.A. Legotin, A.A. Krasnov, V.N. Murashev, Y.K. Omel'chenko, O.I. Rabinovich, A.S. Laryushkin, J. Nano-Electron. Phys. 7 No 4, 04047 (2015).

12. C. Donolato, Solid-State Electron. 31(11), 1587 (1988).

13. E.B. Yakimov, V.V. Privezentsev, J. Mater. Sci: Mater. Electron. 19, 277 (2008).

14. M.A Polikarpov, E.B. Yakimov. Solid State Phenom. 242, 312 (2016). 